\title{
A comparison of the probability distribution of observed substorm magnitude with that predicted by a minimal substorm model
}

\author{
S. K. Morley ${ }^{1,2}$, M. P. Freeman ${ }^{2}$, and E. I. Tanskanen ${ }^{3,4}$ \\ ${ }^{1}$ School of Mathematics and Physical Sciences, University of Newcastle, Callaghan, NSW 2308, Australia \\ ${ }^{2}$ British Antarctic Survey, Natural Environment Research Council, High Cross, Madingley Road, Cambridge, CB3 0ET, UK \\ ${ }^{3}$ Department of Physics and Technology, University of Bergen, Norway \\ ${ }^{4}$ Finnish Meteorological Institute, Space Research Unit, Helsinki, Finland
}

Received: 15 May 2007 - Revised: 1 November 2007 - Accepted: 6 November 2007 - Published: 29 November 2007

\begin{abstract}
We compare the probability distributions of substorm magnetic bay magnitudes from observations and a minimal substorm model. The observed distribution was derived previously and independently using the IL index from the IMAGE magnetometer network. The model distribution is derived from a synthetic AL index time series created using real solar wind data and a minimal substorm model, which was previously shown to reproduce observed substorm waiting times. There are two free parameters in the model which scale the contributions to AL from the directly-driven DP2 electrojet and loading-unloading DP1 electrojet, respectively. In a limited region of the 2-D parameter space of the model, the probability distribution of modelled substorm bay magnitudes is not significantly different to the observed distribution. The ranges of the two parameters giving acceptable (95\% confidence level) agreement are consistent with expectations using results from other studies. The approximately linear relationship between the two free parameters over these ranges implies that the substorm magnitude simply scales linearly with the solar wind power input at the time of substorm onset.
\end{abstract}

Keywords. Ionosphere (Modeling and forcasting) - Magnetosphere physics (Solar wind-magnetosphere interactions; Storms and substorms)

\section{Introduction}

Since the introduction of the substorm concept as a sequence of events observed in the aurora (Akasofu, 1964), much of the focus in the literature has been on the phenomenology of the substorm. From this, a picture has developed in which the substorm has three distinct phases: growth, expansion and recovery (Akasofu, 1964; McPherron et al., 1973). In

Correspondence to: S. K. Morley

(steven.morley@newcastle.edu.au) the growth phase, energy is accumulated in the magnetospheric lobes. Substorm onset and the ensuing expansion phase is marked by the sudden release of this stored energy, the mechanism by which this occurs is still a subject of lively debate (e.g. Baker et al., 1999; Lui, 2001). In the expansion and recovery phases the released energy is dissipated via different channels, including ionospheric Joule heating, ring current enhancement and plasmoid ejection. A number of phenomenological models have been developed that describe the detailed evolution of various observables during the substorm (e.g. Lui, 1991, and references therein). These models agree with the 3-phase cycle but differ in the emphasis they place on describing different observables, especially those associated with substorm onset. Whilst being very useful in synthesizing complicated observations and motivating possible physical descriptions, phenomenological models do not quantify or predict behaviour.

Thus, in tandem with phenomenology, mathematical models have been developed. Global MHD models solve the fundamental physical equations of large-scale plasma dynamics in a realistic magnetospheric geometry but do not adequately capture processes on the kinetic scale that can couple to the large scale. Consequently, they have had limited success in reproducing substorm behaviour (see Raeder and Maynard, 2001, and other papers in that issue). Furthermore, their behaviour can be almost as difficult to understand as the real system and the very long simulations or statistical ensembles that would be necessary to analyse their non-linear dynamics are impractical due to the large computational expense. Intermediate complexity models have been designed that are less computationally expensive and which arguably try to improve the representation of kinetic-scale processes appropriate to the substorm, at the expense of simplified physics and a simplified magnetospheric geometry (Klimas et al., 1992, 1994; Horton and Doxas, 1998; Klimas et al., 2004). The dynamics of these models can be analysed statistically but is complicated by the number of free parameters.

Published by Copernicus Publications on behalf of the European Geosciences Union. 
Consequently, it is also desirable to derive a minimal substorm model which captures the essential properties of substorms with a minimal number of parameters, such that its dynamical properties can be more completely analysed and understood.

Based on principles developed in Freeman and Farrugia (1995, 1999) and consistent with the 3-phase substorm phenomenology, a Minimal Substorm Model (MSM) has been developed with just three mathematical rules and one free parameter (Freeman and Morley, 2004, hereafter F and M). This model is the first to explain the timing of substorms, by reproducing the probability distribution of waiting times between substorms observed by Borovsky et al. (1993). The model focusses on the dynamics of the whole system, without explicitly adopting any of the instabilities proposed to cause substorm onset.

In this paper we present a synthetic AL index comprised of two parts (directly-driven and loading-unloading) derived from basic physical considerations and empirical relationships. The loading-unloading component of the synthetic index is here driven by the MSM. Thus we can test the MSM further by comparing the probability distributions of substorm sizes from the model and from observation. The observed distribution is of substorm magnetic bay magnitudes measured by Tanskanen et al. (2002) using the IL index (Kallio et al., 2000), a local AL index derived from the IMAGE magnetometer network (Viljanen and Häkkinen, 1997) (http://www.ava.fmi.fi/image). Here, we repeat their analysis using the synthetic AL index and compare the results.

\section{Method}

Tanskanen et al. (2002, hereafter TEA) identified substorms from the IL index using certain selection criteria (discussed in Sect. 3.1) and measured substorm size by the peak magnitude of the IL index during the substorm so identified. Thus, in order to compare best their substorm size distribution with that of the MSM, it is desirable to create a synthetic IL index from the minimal substorm model and analyse it in the same way as TEA. This section explains the method for creating the synthetic IL index. F and M give full details of the MSM, with justifications and limitations.

The IL index is a local magnetic index derived from the IMAGE magnetometer network, which mimics the global AL index in the 17:30-04:00 MLT sector (Kauristie et al., 1996; Kallio et al., 2000). It comprises a latitudinal chain of magnetometers and thus is less prone to measurement errors in the magnetic bay magnitude caused by variations in the latitude of the auroral electrojet. Thus, in what follows, we shall use IL and AL interchangeably, but remembering the local time restriction, where necessary.

The AL and other Auroral Electrojet (AE) indices are derived from the northward $(H)$ component of the geomagnetic field vector measured at 12 reference stations situated under the statistical location of the auroral oval (Davis and Sugiura, 1966). The AL index is the 1-min averaged time series formed by the lower envelope of the $H$ component measurements from all stations (i.e. the most negative disturbance), which is induced by westward electrojet equivalent currents.

The AL index consists of two primary components - the DP1 component arising from the unloading process of the substorm and the DP2 component that is directly-driven by the solar wind (e.g. Sun et al., 1998). Thus we write:

$\mathrm{AL}=\mathrm{AL}_{1}+\mathrm{AL}_{2}$

where $\mathrm{AL}_{1}$ and $\mathrm{AL}_{2}$ are the DP1 and DP2 components of $\mathrm{AL}$, respectively.

\subsection{The directly-driven component - $\mathrm{AL}_{2}$}

The directly-driven component of the AL index comes from the DP2 current system (Nishida and Kokubun, 1971) associated with the typically two-cell ionospheric convection cycle of Dungey (1961). Remembering that the AL index is derived from the northward magnetic perturbation $\Delta H$ measured on the ground, we first use the Biot-Savart law to relate this to an eastward equivalent current to get:

$\Delta H=g J_{e}$

where $J_{e}$ is the height-integrated eastward ionospheric equivalent current density above the magnetometer location and $g$ is a geometrical factor that depends on the horizontal variation of $J_{e}$ in the vicinity. For example, $g=\mu_{0} / 2$ for an infinite plane current sheet.

In the approximation of a vertical magnetic field and uniform ionospheric conductivity, the equivalent current is equal to the Hall current (Fukushima, 1969)

$J_{e}= \pm \Sigma_{H} E_{n}$

where $\Sigma_{H}$ is the height-integrated Hall conductivity and $E_{n}$ is the northward component of the electric field. The \pm -signs refer to the northern/southern hemisphere.

The electric field at any point in the ionosphere can be expressed in terms of the total electric potential drop $V_{\max }(>0)$ associated with the convection pattern (which measures the total rate of magnetic flux transport)

$E_{n}=f V_{\max }$

Here $f$ is a factor that depends on position $(r, \lambda, \phi)$ and the assumed ionospheric convection pattern. $r, \lambda$ and $\phi$ are the radial, latitudinal and azimuthal coordinates, respectively. For example, from the ionospheric convection solution given in Freeman et al. (1991) and Freeman (2003) we have

$f=\frac{-1}{r \cos \lambda} \sum_{m=1}^{\infty} m c_{m} \sin (m \phi) \frac{\cosh \left(m\left(x-x_{2}\right)\right)}{\sinh \left(m\left(x_{1}-x_{2}\right)\right)}$

where $x=\log _{e}(\tan (\pi / 4-\lambda / 2))$ and the equation applies to auroral latitudes between the region 1 current system at $x=x_{1}$ 
and the region 2 current system at $x=x_{2}$. In this model of the Dungey (1961) paradigm, the locus $x=x_{1}$ is co-located with the region 1 current, the convection reversal boundary, and the boundary between open and closed geomagnetic field lines, commonly referred to as the polar cap boundary. $c_{m}$ is the coefficient of a Fourier series expansion of the electric potential distribution around the polar cap boundary such that

$c_{m}=-\frac{1}{2}$ for $\quad m=1 \quad c_{m}=0$ for $m>1$

for the simplest ionospheric convection pattern. In this case, the transport of plasma and magnetic flux into the polar cap across the dayside polar cap boundary is balanced by the plasma and flux transport out of the polar cap across the nightside polar cap boundary, such that the polar cap does not expand. This may be appropriate to the late expansion phase of the substorm when the peak AL magnitude is realized (Lockwood and Cowley, 1992). Typically, the axis of symmetry of the 2-cell convection pattern is observed to be at an angle $\pi / 12<\phi_{s}<\pi / 6$ counter-clockwise with respect to the noon-midnight meridian (e.g. Ruohoniemi and Greenwald, 1996). Thus we may write $\phi=\phi^{\prime}+\phi_{s}$, where $\phi^{\prime}$ is the angular equivalent of MLT and $\phi^{\prime}=0$ corresponds to 0 MLT.

Many studies have shown good correlation between various functions of solar wind variables and the transpolar voltage $V_{\max }$. Reiff et al. (1981) found the highest correlations were between a modified form of the $\varepsilon$ function (Perreault and Akasofu, 1978) and linear or quadratic powers of $V_{\max }$. Using emu units, Perreault and Akasofu defined $\varepsilon=L_{0}^{2}\left(v B^{2} / 4 \pi\right) \sin ^{4}(\theta / 2)$, where $v$ is the solar wind speed, $B$ is the magnitude of the interplanetary magnetic field (IMF), $\theta$ is the IMF clock angle, and $L_{0}$ is a length scale estimated to be $7 R_{E}$. Defining $\varepsilon=P A_{\text {eff }}$ and using SI units we have a power input per unit area

$P=\left(v B^{2} / \mu_{0}\right) \sin ^{4}(\theta / 2)$

where $\mu_{0}$ is the permeability of free space, and an effective area $A_{\text {eff }}=4 \pi L_{0}^{2}$ (Koskinen and Tanskanen, 2002). Applying the Reiff et al. modification, $P$ becomes

$P_{a l}=\left(v B_{a l}^{2} / \mu_{0}\right) \sin ^{4}(\theta / 2)$

where $B_{a l}$ is an amplified-limited form of the IMF magnitude $B$ given by

$B_{a l}[\mathrm{nT}]=\min \left(\mathrm{B}[\mathrm{nT}], \frac{60[\mathrm{nT}]}{\mathrm{F}}\right)$

where we take the amplification factor $F$ to be 7 (Reiff et al., 1981). This is thought to represent the effects of IMF compression in the dayside magnetosheath on the solar wind power input, $P$.

We have determined a polynomial equation for $V_{\max }$ that lies between the linear and quadratic relations found empirically by Reiff et al.:

$V_{\max }[k V]=20 \sqrt[3]{\pi P_{a l}\left[\mu \mathrm{W} \mathrm{m}^{-2}\right]}+1.4 \pi P_{a l}\left[\mu \mathrm{W} \mathrm{m}^{-2}\right]$
Unlike their quadratic relationship, this equation has a real potential drop for any solar wind input, but, unlike their linear relationship, passes through the origin (thus neglecting any contribution to the transpolar voltage from viscous interaction). Through the amplification-limitation procedure of Eq. (9), $V_{\max }$ saturates at about $200-250 \mathrm{kV}$ for a high magnetic field strength (depending on the solar wind velocity), in agreement with observation (e.g. Russell et al., 2001; Hairston et al., 2003), theoretical prediction (Hill et al., 1976) and MHD modelling (see Siscoe et al., 2004, for a review). The commonly-used linear relationship does not display this saturation characteristic to the same extent and for similar values of the solar wind variables predicts polar cap voltages of over $300 \mathrm{kV}$.

Combining Eqs. (2), (3), (4) and (10), we get

$\mathrm{AL}_{2}=\Delta H= \pm f g \Sigma_{H} V_{\max }=-a V_{\max }\left(P_{a l}\right)$

where $a$ has the same sign at conjugate points in both hemispheres by construction and is expected to be positive by the requirement that $A L<0$. To synthesize the $A L$ index, we note that the relevant contributing magnetometers are localized in latitude but distributed in MLT (Davis and Sugiura, 1966). Thus, $a$ would be evaluated at the typical latitude $\lambda$ of the contributing AL stations and at the MLT $\left(\phi^{\prime}\right)$ giving the minimum AL. From Eq. (1), this equation is evaluated at the $\mathrm{AE}$ magnetometer station giving the minimum $\mathrm{AL}$ value. Generally, this may not be the location where $\mathrm{AL}_{2}$ itself is a minimum, in that $\mathrm{AL}_{2}$ is expected to minimise just prior to dawn MLT (i.e. $\phi=\pi / 2$ ), whereas $\mathrm{AL}_{1}$ will likely minimise in the DP1 electrojet region around midnight MLT. Empirically, AL comes mostly from magnetometers located in the 00:00-06:00 MLT sector (Allen and Kroehl, 1975).

In contrast, to synthesize the IL index, we should remember that it is compiled from a magnetometer network that is distributed in latitude and localized in MLT. Thus, $a$ would be evaluated at the typical MLT $\left(\phi^{\prime}\right)$ of the contributing IL stations (which varies with Universal Time) and at the latitude $\lambda$ giving the minimum IL. Furthermore, $\phi^{\prime}$ should be restricted only to the range 17:30-04:00 MLT for which IL is valid as a suitable alternative to AL (Kauristie et al., 1996).

For simplicity, in what follows we shall assume $a$ to be constant in time and find the value of $a$ that yields the best agreement between the probability distributions of modelled and observed substorm magnetic bay magnitudes. In order to aid the interpretation of this value of $a$, let us first estimate its likely bounds. Since the substorm magnetic bay magnitude is equal to the minimum value of the synthetic IL index during the expansion phase it is reasonable to assume that: (a) the contributing station is between 20:29 MLT and 01:15 MLT (the \pm 1 standard deviation limits of the probability distribution of onset MLT for the TEA substorms) and at $\lambda=70^{\circ}$ (the most common magnetic latitude of the minimum IL for the TEA substorms); (b) the polar cap boundary is at the same magnetic latitude, in order that the contributing station is at the latitude where the eastward equivalent current minimises 
in the $\mathrm{AL}_{2}$ model; and (c) the height-integrated Hall conductivity $\Sigma_{H}=15$ Siemens, as measured in the midnight MLT sector during the expansion phase of an individual substorm (Coumans et al., 2004) and comparable to the conductivity measured for a statistical ensemble of 28 isolated substorms when averaged over the ensemble and over the whole substorm region (Gjerloev and Hoffman, 2000). Let us also assume that: (d) the simple convection pattern associated with Eqs. (5) and (6) with $\phi_{s}=\pi / 12$ and with the region 2 current system at a magnetic latitude of $55^{\circ}$; (e) the Hall current layer is at a height of $110 \mathrm{~km}$ above the Earth $(\mathrm{r}=6370+110 \mathrm{~km})$; and $(f) g=\mu_{0} / 2$. Then we get $a<2.3 \times 10^{-12} \mathrm{~T} \mathrm{~V}^{-1}$. Thus, for $P=8 \times 10^{-6} \mathrm{~W} \mathrm{~m}^{-2}$ (i.e. corresponding to a solar wind speed of $400 \mathrm{~km} \mathrm{~s}^{-1}$ and a southward IMF of $5 \mathrm{nT}$ ) we have $V_{\max }=94 \mathrm{kV}$ and $\mathrm{AL}_{2}>-212 \mathrm{nT}$.

\subsection{The Unloading Component $-\mathrm{AL}_{1}$}

The unloading component of the AL index comes from the DP1 current system (Nishida and Kokubun, 1971) associated with the substorm current wedge (McPherron et al., 1973). Using a superposed epoch analysis of the AE indices for an ensemble of isolated substorms, Weimer (1994) showed that the average AL curves, from substorm onset at time $t=0$ to the end of the recovery phase, fitted the function

$$
-\langle\mathrm{AL}\rangle=c+b t \exp (p t)
$$

where the time constant $p=-2.22 \mathrm{~h}^{-1}$ for substorms of medium intensity and \langle\rangle denotes the ensemble average. From the symmetry of the AU and AL indices, Weimer (1994) argued that the $c$ and $b$ terms represented the ensemble averages of the directly-driven component $\mathrm{AL}_{2}$ and the unloading component $\mathrm{AL}_{1}$, respectively. Thus, the contribution to $\mathrm{AL}_{1}$ from the $i$ th substorm of the ensemble is

$$
\mathrm{AL}_{1 i}=-b_{i} S\left(t-t_{i}\right) \text { for } t>t_{i}
$$

where $t_{i}$ is the substorm onset time and we have assumed that each substorm has the same functional form $S(t)=t \exp (p t)$ but variable amplitude $b_{i}$.

The amplitude $b_{i}$ can be expressed in terms of the total Joule heating energy dissipated in the northern hemisphere ionosphere $W_{J i}$ using an empirical linear relationship deduced by Ahn et al. (1983) between hemispheric Joule heating and the AL index. Integrating Eq. (13) from time $t=t_{i}$ to $t=\infty$ using this relationship we have

$$
\begin{array}{r}
W_{J i}=-\alpha \int_{t_{i}}^{\infty} \mathrm{AL} \mathrm{d} t \\
=\frac{\alpha b_{i}}{p^{2}}
\end{array}
$$

where $\alpha=3 \times 10^{8} \mathrm{~W} \mathrm{nT}^{-1}$.

Joule heating represents only a fraction $\beta$ of the total energy released by unloading $W_{i}$. The energy is distributed among four main sinks: Joule heating, particle precipitation, ring current enhancement, and plasmoid ejection (including associated plasma sheet heating, Ieda et al., 1997). To a first approximation, Joule heating can be considered to account for one third of the total energy budget (Kamide and Baumjohann, 1993; Tanskanen et al., 2002). If we make a further assumption that Joule heating is equally divided between the northern and southern hemispheres then $\beta \sim 1 / 6$.

Thus we now re-express Eq. (13) as

$$
\mathrm{AL}_{1 i}=-\frac{\beta p^{2}}{\alpha} W_{i} S\left(t-t_{i}\right)
$$

\subsection{The minimal substorm model}

Equation (15) represents the unloading component for a single isolated substorm. In order to create a sequence of substorms, we use the F\&M MSM (see also Freeman and Farrugia, 1995, 1999). The model predicts the onset time $t_{i}$ and total energy $W_{i}$ of the $i$ th substorm based on just three mathematical rules and an empirical solar wind power input $P$.

In the MSM, the total energy released in each substorm, $W_{i}$, is given by

$$
W_{i}=A_{\mathrm{eff}} D P\left(t_{i}\right)
$$

where $A_{\text {eff }}$ is the effective area of the magnetopause for solar wind power input (see Sect. 2.1). Defining $A_{\text {eff }}=L_{0}^{2}$ and using emu units, Perreault and Akasofu (1978) estimated $L_{0}=7 R_{E}$. Converting into the SI units we use here, we have $A_{\text {eff }}=4 \pi L_{0}^{2}$ (see Koskinen and Tanskanen, 2002). Consequently, the Perreault and Akasofu (1978) estimate would give $A_{\text {eff }}=196 \pi R_{E}^{2}$. However, Koskinen and Tanskanen (2002) have suggested that, though the coupling efficiency may vary, an increased value of about $L_{0}=10 R_{E}$ fitted better with their observations, which would give $A_{\mathrm{eff}}=400 \pi R_{E}^{2}$

Referring to the MSM rules given by F and M, it should be noted that in the present study, the amplified-limited power input, $P_{a l}$, has been used in rule 1 to determine the energy flux into the magnetosphere because it is thought to represent the effects of IMF compression in the dayside magnetosheath on energy input through magnetopause reconnection (Reiff et al., 1981). However, amplification-limitation has not been used in rule 2 to determine the magnetospheric ground state because we view this state to depend on the magnetotail boundary condition where the magnetosheath conditions are more similar to those of the IMF (i.e. we use $P$ in Eq. 16).

$D$ is a constant equal to the substorm recurrence period for constant $P$. F\&M derived the value of $D=2.69 \mathrm{~h}$ by comparing an observed probability distribution of substorm waiting times with that of the MSM driven by real solar wind data, but without applying the amplification-limitation procedure described previously. Implementing the procedure, and redoing the analysis as before, yields a slightly higher best-fit value of $D=2.73 \mathrm{~h}$. 


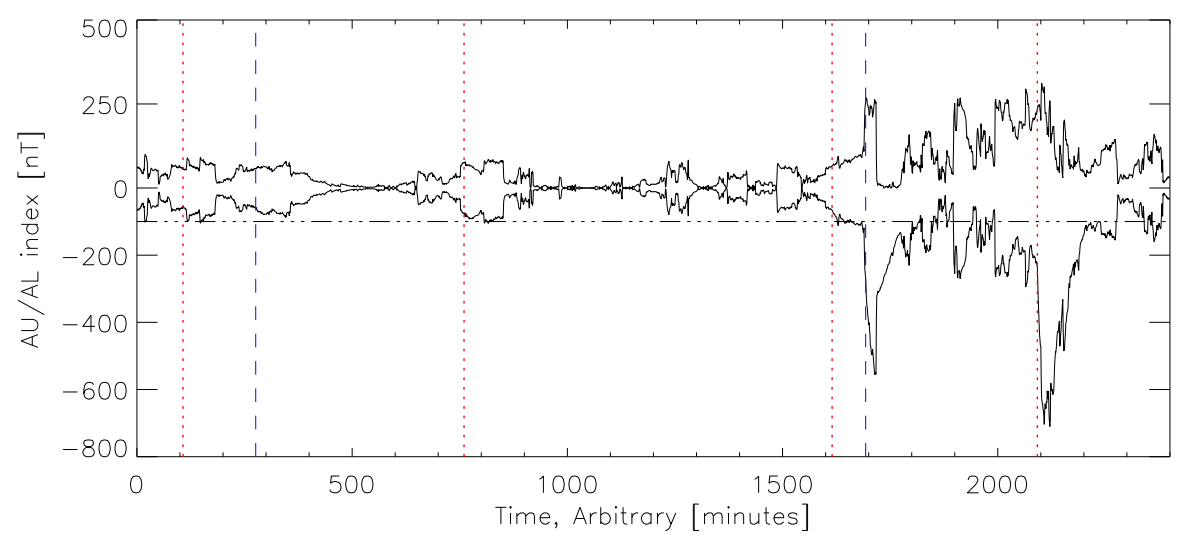

Fig. 1. An example section of the simulated AL index. The directly driven component has been inverted and plotted to represent the AU index. The horizontal black dot-dashed line marks the $-100 \mathrm{nT}$ threshold level; the vertical red dotted lines mark substorm onsets that are counted after grouping and application of the threshold $(t=(107,760,1615,2092)$ min $)$; the vertical blue dashed lines mark substorm onsets that are excluded $(t=(276,1693) \mathrm{min})$. See text for further details.

Substituting Eq. (16) into Eq. (15) and summing over all substorms, we get

$\mathrm{AL}_{1}(t)=-\sum_{i=1}^{N} k P\left(t_{i}\right) H\left(t-t_{i}\right) S\left(t-t_{i}\right)$

where $H$ is the Heaviside step function and the parameter $k=\beta p^{2} A_{\text {eff }} D / \alpha$. Taking $\beta=1 / 6, \quad p=-2.22 \mathrm{~h}^{-1}$, $L_{0}=7 R_{E}, \quad D=2.73 \mathrm{~h}$, and $\alpha=3 \times 10^{17} \mathrm{~W} \mathrm{~T}^{-1}$, we have $k=5.2 \times 10^{-5} \mathrm{~m}^{2} \mathrm{~T} \mathrm{~J}^{-1}$. Neglecting contributions from neighbouring substorms, the peak $\mathrm{AL}_{1}$ magnitude occurs at $t-t_{i}=-1 / p$ where $\mathrm{AL}_{1 p}=k P /(p e)$. For $P=8 \times 10^{-6} \mathrm{~W} \mathrm{~m}^{-2}$, which corresponds to a solar wind speed of $400 \mathrm{~km} \mathrm{~s}^{-1}$ and a southward IMF of $5 \mathrm{nT}$, we find that $\mathrm{AL}_{1 p}=-250 \mathrm{nT}$.

\subsection{The Synthetic AL}

Substituting Eqs. (17) and (11) into Eq. (1), we get the following expression for the complete AL index

$\mathrm{AL}(t)=-k \sum_{i=1}^{N} P\left(t_{i}\right) H\left(t-t_{i}\right) S\left(t-t_{i}\right)-a V_{\max }\left(P_{a l}(t)\right)$

Thus we have two free parameters in the AL model: $k$, which scales the amplitude of the unloading DP1 component; and $a$, which scales the amplitude of the directly-driven DP2 component. We have estimated $a<2.3 \times 10^{-12} \mathrm{~T} \mathrm{~V}^{-1}$ and $k=5.2 \times 10^{-5} \mathrm{~m}^{2} \mathrm{~T} \mathrm{~J}^{-1}$, but it is important to remember that this is a rough approximation. Thus, in the subsequent analysis, we explore the goodness-of-fit of our model results to the observations for a range of $\{a, k\}$ parameter space.

\section{Analysis and results}

Following the above method, a synthetic AL index was generated using solar wind measurements from the NASA
Wind spacecraft between 1 January 1995 and 1 July 1998 (see $\mathrm{F}$ and $\mathrm{M}$ for details). Only sections of solar wind data that were unbroken for $100 \mathrm{~h}$ or more were used, of which there were 37 . An example section of the modelled $\mathrm{AL}$ index is shown in Fig. 1 for $k=5.19 \times 10^{-5} \mathrm{~m}^{2} \mathrm{~T} \mathrm{~J}^{-1}$, $a=1.45 \times 10^{-12} \mathrm{~T} \mathrm{~V}^{-1}$ and $D=2.73 \mathrm{~h}$. The substorm onset times are marked by the vertical dotted and dashed lines at $t=[107,276,760,1615,1693,2092] \mathrm{min}$. Like the real AL time series, the model yields a complicated time series with substorm magnetic bays of widely-varying magnitude and structure. In the model, substorm onset is also typically preceded by an interval of about an hour during which the DP2 component of $\mathrm{AU}$ and $-\mathrm{AL}$ is relatively enhanced, similar to the growth phase signature observed in real auroral magnetic records (McPherron et al., 1973).

\subsection{Substorm identification}

TEA defined threshold and grouping criteria to identify a substorm from their IL data: A substorm was defined as a magnetic bay signature whose magnitude exceeds $100 \mathrm{nT}$ and for which the time elapsed between the peak of a substorm and the onset of the following substorm must exceed $2 \mathrm{hrs}$, otherwise the substorms are grouped and treated as a single event. Through 1997 and 1999 they identified $N_{a}=827$ "admissible" substorms. The probability distribution of the MLT of substorm onset was approximately Gaussian with mean $=22.9 \mathrm{MLT}$ and standard deviation $\sigma=2.4 \mathrm{~h}$. Assuming that this distribution reflects the substorm detection efficiency using the local IL index, with perfect detection at the mean MLT, and that substorms are uniformly distributed in UT, then the total number of substorms is estimated to be $24 N_{a} /(\sigma \sqrt{2 \pi})=3321$, and the average substorm waiting time is inferred to be $5.3 \mathrm{~h}$. This is similar to the mean substorm waiting time of $5.7 \mathrm{~h}$ found independently by Borovsky et al. (1993) for 1001 substorm pairs in 1982-1983. 

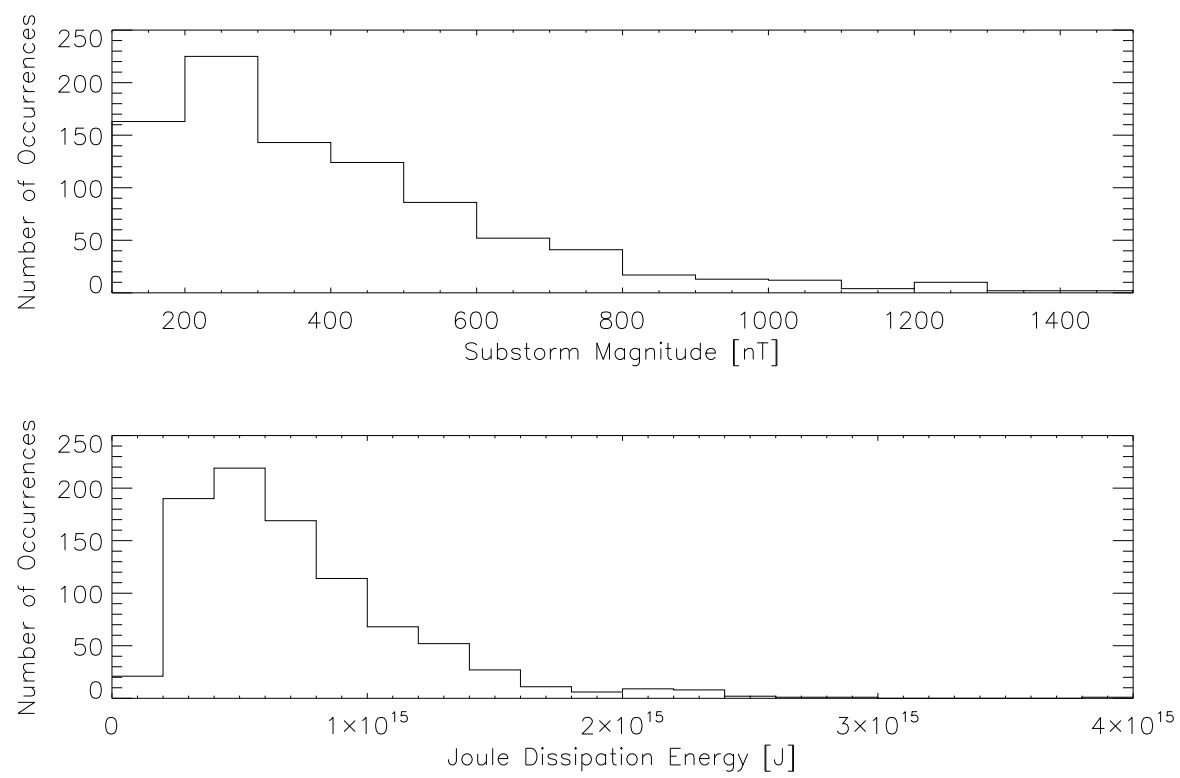

Fig. 2. Substorm magnitude (upper panel) and Joule dissipation energy (lower panel) histograms derived from the synthetic AL index for the model set of substorms. The histograms are binned by every $100 \mathrm{nT}$ in substorm magnitude and by every $2 \times 10^{14} \mathrm{~J}$ in Joule dissipation energy. Compare to Fig. 5 of TEA.

We have applied the same threshold and grouping criteria as TEA to the substorms contained in the synthetic AL index. In the example shown in Fig. 1, the substorm onsets identified in this way can be seen at times $t=(107,760,1615$, 2092) min, marked by the vertical red dotted lines. The substorm at $t=276 \mathrm{~min}$ has a peak magnitude below the threshold level and is therefore excluded. The substorm at $t=1693 \mathrm{~min}$ starts $78 \mathrm{~min}$ after the previous onset and is therefore grouped with the substorm at $t=1615 \mathrm{~min}$. Using the threshold and grouping criteria the number of "admissible" substorms is 718 in 5258 data hours, corresponding to an average substorm occurrence rate of approximately $7.3 \mathrm{~h}$.

This is considerably longer than the TEA mean waiting time of $5.3 \mathrm{~h}$. Applying a shorter grouping window than stated in TEA decreases the average waiting time. In fact, in the original model time series (without grouping) 932 substorm onsets occurred, corresponding to a mean substorm waiting time of $5.7 \mathrm{~h}$. Inspection of the TEA data set shows that where substorms were of shorter duration, the grouping criterion was not strictly applied. In many cases, this reduced the inter-substorm interval to about 1 hour. For example, reducing the grouping window to $27 \mathrm{~min}$ (i.e. if onset occurs during the expansion phase of the previous substorm then the substorms are grouped) gives 904 modelled substorms in $5258 \mathrm{~h}$, a mean inter-substorm interval of $5.8 \mathrm{~h}$. This compares much better to the Borovsky et al. data set mean waiting time of $5.7 \mathrm{~h}$ used to define the $D$ parameter of the MSM.

In the subsequent analysis we adopt the TEA grouping criterion, but also examine the effect of changing the grouping window on the distribution of substorm magnitudes.

\subsection{Substorm bay magnitudes}

For each substorm identified under the TEA criteria, the peak magnitude of the simulated AL index was found, and the total energy dissipated by Joule heating was calculated using the first line of Eq. (14) with the upper integration limit replaced by the end time of the substorm, taken here to be $3 \mathrm{~h}$ after the onset. In the case of multiple substorms being grouped, the end time is $3 \mathrm{~h}$ after the onset of the last substorm in the group. Figure 2 shows the occurrence distribution of these substorm magnitudes and Joule dissipation energy for the same choice of the model free parameters $\{a, k\}$ that was used in Fig. 1. These distributions can be compared to the upper and lower panels of Figs. 5 and 7 of TEA, which show the equivalent observed distributions for isolated and stormtime substorms separately. The model distributions are similar to those presented by TEA, if the isolated and stormtime substorms are considered together. For example, the tail of the model distribution cannot be accounted for by isolated substorms alone.

\subsection{Statistical tests}

The null hypothesis that the observed and simulated distributions of substorm AL magnitude come from the same distribution was tested over a range of $\{a, k\}$ values using two different test statistics. Figure 3 shows contours of constant test statistic over the $\{a, k\}$ parameter space for the two-sided Kolmogorov-Smirnov (K-S) test (left panel) and the twosample $\chi^{2}$ test (right panel) (e.g. Conover, 1999). 

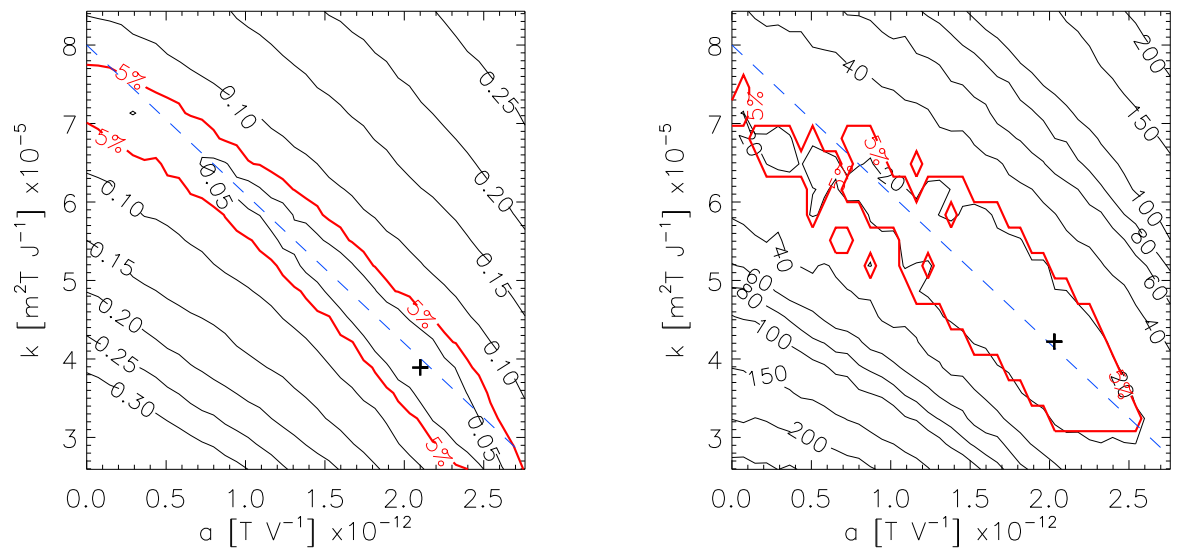

Fig. 3. Contour maps showing (on the left) the Kolmogorov-Smirnov test statistic and (on the right) the $\chi^{2}$ test statistic for $\{a, k\}$ parameter space. The thick red contours indicate the $5 \%$ significance level. The point of best fit is marked by a cross. The dashed blue line is the linear equation $k=s a-p e d$, for $s=2 \times 10^{7} \mathrm{~m}^{2} \mathrm{C}^{-1}$, ped $=-8 \times 10^{-5} \mathrm{~m}^{2} \mathrm{~T} \mathrm{~J}^{-1}$, as described in Sect. 4.1

The two-sample $\chi^{2}$ test (e.g. Conover, 1999) requires that the data be sorted into bins and is more sensitive to differences in the bins with low observed frequencies (i.e. those bins in the tail of the distribution). The data were binned in $100 \mathrm{nT}$ intervals and neighbouring bins were joined where the expected frequencies were less than five. At the optimal values of $a$ and $k$ (indicated by a cross in the figure) this resulted in 13 classes and hence 12 degrees of freedom. The calculated value of $\chi^{2}=8.06$ is less than the corresponding $\chi^{2}=21.03$ at the $5 \%$ significance level and allows us to accept the null hypothesis for the optimal values of $a=2.03 \times 10^{-12} \mathrm{~T} \mathrm{~V}^{-1}$ and $k=4.22 \times 10^{-5} \mathrm{~m}^{2} \mathrm{~T} \mathrm{~J}^{-1}$.

The K-S test is based on the maximum difference between the cumulative distribution functions of the two samples. Hence it is more sensitive to differences near the median of the distribution. For the optimal fit at $a=2.10 \times 10^{-12} \mathrm{~T} \mathrm{~V}^{-1}, k=3.89 \times 10^{-5} \mathrm{~m}^{2} \mathrm{~T} \mathrm{~J}^{-1}$, the K-S parameter $s=0.025$, which is also less than the $5 \%$ significance level $(s=0.069)$.

The 5\% significance level is marked in each plot by a heavy red line. At this level, the model distribution of substorm magnitude is found to be drawn from the same distribution as the observed distribution for a wide range of parameters $a$ and $k$, but there is a clear relationship between the two parameters. As the $a$ contribution to AL from the directly-driven component is scaled down, the $k$ contribution from unloading necessarily increases, thus preserving the substorm AL magnitude. Averaging the best fit parameters of the two tests gives overall best fit parameters of $k=4.05 \times 10^{-5} \mathrm{~m}^{2} \mathrm{~T} \mathrm{~J}^{-1}$ and $a=2.07 \times 10^{-12} \mathrm{~T} \mathrm{~V}^{-1}$.

For these parameters, the model and observed distributions of substorm AL magnitude are shown in Fig. 4. The observational data includes both "isolated" and "stormtime" substorms, as defined by TEA. The distribution of modelled substorm AL magnitudes is shown by the solid red line and the distribution measured by TEA is shown by the solid black line. The occurrence frequency of modelled magnitudes has been normalized to the number of substorms in the observational data set. The good agreement can be clearly seen. For comparison, the blue dashed line shows the distribution of modelled substorm magnitudes using a 27 min grouping window (see Sect. 3.1). It can be seen that this distribution is similar to that obtained using the TEA grouping and threshold criteria. The light blue dotted line shows the MSM substorm magnitude distribution with no grouping applied.

\section{Discussion}

\subsection{Substorm magnetic bay magnitudes}

We have shown that the distribution of substorm magnetic bay magnitudes derived from the IL index by TEA is not significantly different to that derived from a synthetic AL index based on the MSM and using similar substorm threshold and grouping criteria.

Apart from the single free parameter $D$ of the MSM, which is fixed by the distribution of substorm waiting times (Freeman and Morley, 2004), two free parameters, $a$ and $k$, are used in the construction of the synthetic AL index, and hence in the derivation of the substorm magnitudes. Parameter $a$ scales the amplitude of the directly-driven $D P 2$ component and parameter $k$ scales the amplitude of the unloading $D P 1$ component. The relative importance of these parameters to the substorm AL magnitude can be understood from Fig. 3, which shows how the similarity of the observed and modelled distributions of substorm AL magnitude varies for different combinations of $a$ and $k$.

Considering the 5\% significance level, highlighted in the figure by the bold curve, the model is able to account for the observed distribution of substorm AL magnitudes over 


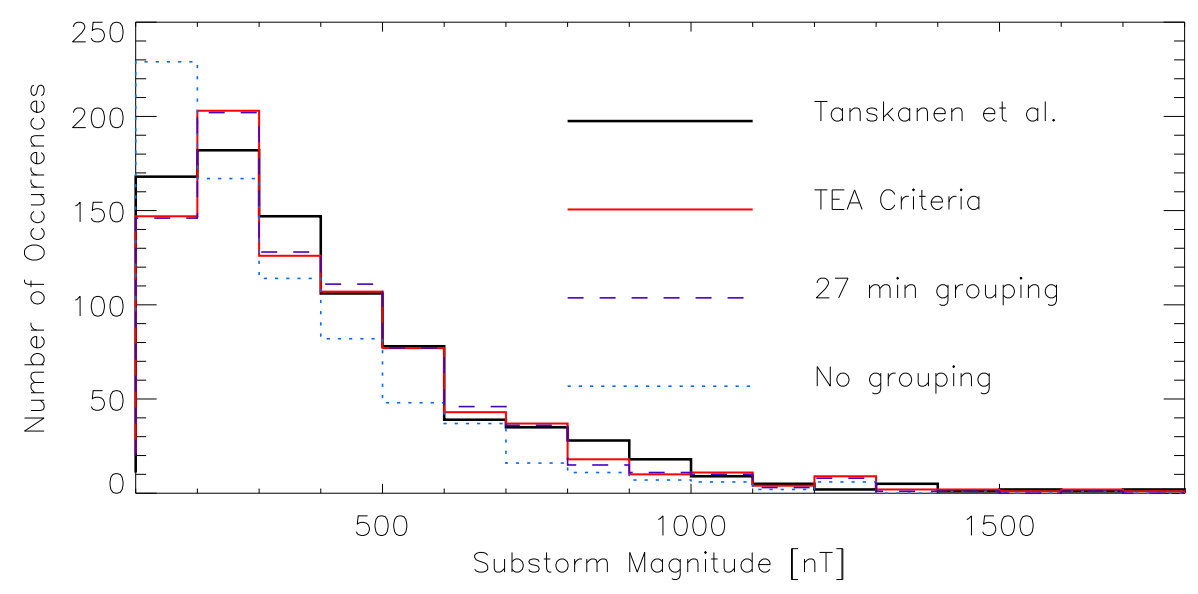

Fig. 4. A histogram of substorm magnitude as measured using the IL index by TEA (thick black line) and using the synthetic AL index derived from the minimal substorm model using the TEA grouping and threshold criteria (solid red line). For comparison the modelled distributions using a shortened grouping criterion (dark blue dashed line) and no grouping criterion (light blue dotted line) are included. Note that the $\mathrm{x}$-axis starts at $100 \mathrm{nT}$.

an approximately linear region of $\{a, k\}$ space. This can be understood from Eq. (18). Neglecting the effect of the TEA substorm grouping and of overlapping substorms, we have that:

$$
\mathrm{AL}_{p i} \approx k S(-1 / p) P\left(t_{i}\right)+a V_{\max }\left(P_{a l}\left(t_{i}-1 / p\right)\right)
$$

where $\mathrm{AL}_{p i}$ is the peak value of $-\mathrm{AL}$ at the end of the $i$ substorm expansion phase at time $t_{i}-1 / p$. In the approximation that $V_{\max }=h P_{a l}(h=$ constant $)$ and $\left(P_{a l}\left(t_{i}-1 / p\right)\right)=P\left(t_{i}\right)$ then

$\mathrm{AL}_{p i} \approx\left(h a-\frac{k}{p e}\right) P\left(t_{i}\right)=d P\left(t_{i}\right)$

where $d=$ constant. Thus the model will yield the same distribution of $\mathrm{AL}_{p i}$, with equally good fit to the observed distribution, for any $\{a, k\}$ satisfying the linear equation $k=s a-p e d$, where $s=p e h$. In Fig. 3, we show this line for $s=-2 \times 10^{7} \mathrm{~m}^{2} \mathrm{C}^{-1}$, ped $=-8 \times 10^{-5} \mathrm{~m}^{2} \mathrm{~T} \mathrm{~J}^{-1}$, which is a good fit to the $5 \%$ plateau for $0.8<a<2.4 \times 10^{-12} \mathrm{~T} \mathrm{~V}^{-1}$. The approximation may be justified as follows: (a) From a superposed epoch analysis of substorms, the IMF is preferentially southward at substorm onset which, for a typical $5 \mathrm{nT}$ IMF and a solar wind speed of $400 \mathrm{~km} / \mathrm{s}$, implies $2<P_{a l}<8 \mu \mathrm{W} \mathrm{m}^{-2}$. Plotting Eq. (10) over this range (not shown), we find that $V_{\max }$ is approximately proportional to $P_{a l}$ with $h \approx 94 \mathrm{kV} / 8 \mu \mathrm{W} \mathrm{m}^{-2}$ (see end of Sect. 2.1)and hence $s=-2 \times 10^{7} \mathrm{~m}^{2} \mathrm{C}^{-1}$. (b) Furthermore, the average north-south component of the IMF changes relatively little during the expansion phase $(\approx 25 \%$ on average) and hence $P_{a l}\left(t_{i}-1 / p\right) \sim P_{a l}\left(t_{i}\right)$. (c) For these (and most) IMF conditions, we have $B \leq 60 / F=8.6 \mathrm{nT}$, and hence $P_{a l}=P$ from Eq. (9). Consequently, in this approximation, the two free parameters reduce to just one free parameter $d$ and the peak amplitude of a substorm is simply proportional to the solar wind power input at the time of substorm onset such that $\mathrm{AL}_{p i}[\mathrm{nT}] \approx 48 P\left(t_{i}\right)\left[\mu \mathrm{W} \mathrm{m}^{-2}\right]$.

The range of parameter $a$ that gives acceptable fits to the observed distribution of substorm magnitudes at the $5 \%$ level matches the expected range of $0<a<2.3 \times 10^{-12} \mathrm{~T} \mathrm{~V}^{-1}$ given at the end of Sect. 2.1, based on the bulk of the TEA substorms. Interestingly, an acceptable fit is found even in the absence of the directly-driven $D P 2$ component $(a=0)$. However, the best fit is obtained for $a \approx 2.1 \times 10^{-12} \mathrm{~T} \mathrm{~V}^{-1}$, emphasizing the general necessity of a directly-driven component of AL, for which there is other compelling evidence (e.g. Weimer, 1994; Sun et al., 1998). The unloading component also appears to be essential (i.e. $k>0$ ), and so the substorm cannot be explained by the linear directly-driven process alone as has been claimed (Akasofu, 1981). The range of acceptable $k$ also includes the value of $k=5.2 \times 10^{-5} \mathrm{~m}^{2} \mathrm{~T} / \mathrm{J}$ estimated at the end of Sect. 2.3, but the best fit value of $k$ (marked by a cross) is lower than this estimate. This could mean that our estimate of the partitioning of unloading energy $(\beta)$ is too high, or that the coupling efficiency $\left(A_{\text {eff }}\right)$ is lower than believed. The former is more likely because Koskinen and Tanskanen (2002) argue that $A_{\text {eff }}$ should, if anything, be higher. Overall, the MSM explains the distribution of substorm bay magnitudes for reasonable values of $a$ and $k$.

\subsection{Energy input and output}

TEA also investigated the relationship between energy input and energy output over different phases of the substorm. They found that the best linear relationship was between the energy input over the expansion phase and the energy output over the same phase. $\mathrm{F}$ and $\mathrm{M}$ pointed out that, in the limit that the expansion phase duration $(\sim 30 \mathrm{~min})$ was short compared to the substorm recurrence time $(\sim 3 \mathrm{~h})$, the empirical 


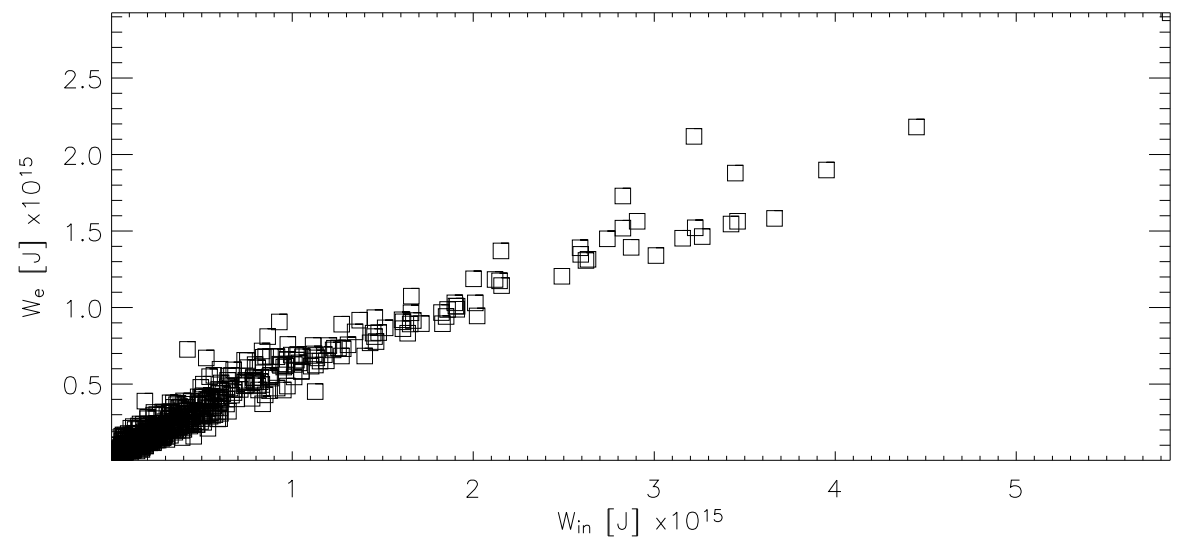

Fig. 5. Joule heating dissipation, $W_{e}$, for modelled substorms as a function of solar wind energy input, $W_{i n}$, during the expansion phase. Compare to Fig. 6a of Tanskanen et al. (2002).

linear relationship was consistent with the assumption of the MSM that the total substorm energy loss is defined to be proportional to the solar wind power input at the time of substorm onset (see also Morley and Freeman, 2007).

Using the synthetic AL index, we can explore this relationship further for non-zero expansion phase duration. Using the best fit parameters of $a$ and $k$ we integrate both the solar wind power input $P_{a l}$ and the ionospheric Joule heating power output derived from the synthetic AL over the expansion phase of each substorm of the MSM. The expansion phase is defined as the time from substorm onset $\left(t=t_{i}\right)$ to peak $-\mathrm{AL}\left(t_{p}=t_{i}-1 / p\right)$. Figure 5 shows the resulting relationship between the modelled energy input and output during the expansion phase. It shows a linear dependence with slope $\sim 0.5$, similar to the linear relationship found by TEA but with a slope of $\sim 0.3$. The energy dissipated by Joule heating can therefore be said to account for about half of solar wind energy input during the substorm expansion phase in the model, compared to the $30 \%$ reported by TEA for isolated substorms. This discrepancy could result from an overestimate of $\beta$ or an underestimate of $A_{\text {eff. The former is more }}$ likely, as discussed in the previous section.

The value of the gradient can be understood as follows: Similar to Eq. (14), the energy dissipated by Joule heating in the northern hemisphere ionosphere during the expansion phase is

$W_{e}=-\alpha \int_{0}^{t_{p}} \mathrm{AL} d t$

Substituting for AL using Eq. (18) and neglecting contributions from neighbouring substorms, we get

$W_{e}=\alpha k \int_{0}^{t_{p}} P\left(t_{i}\right) S(t) d t+\alpha a \int_{0}^{t_{p}} V_{\max }\left(P_{a l}(t)\right) d t$

In the approximation $V_{\max }=h P_{a l}$ and $P_{a l}=P$ (see Sect. 4.1) we get

$W_{e}=\alpha k \int_{0}^{t_{p}} P\left(t_{i}\right) S(t) d t+\alpha a h \int_{0}^{t_{p}} P(t) d t$
Finally noting that the solar wind energy input over the expansion phase is given by $W_{i n}=A_{\text {eff }} \int_{0}^{t_{p}} P_{a l}(t) d t$, we find that

$W_{e}=\frac{-\alpha k}{p A_{\mathrm{eff}}}\left(1-\frac{2}{e}\right) W_{i n}+\frac{\alpha a h}{A_{\mathrm{eff}}} W_{i n}$

and approximating $P_{a l}(t)=P(t)=P\left(t_{i}\right)$ during the expansion phase (see Sect. 4.1). Using the values estimated in Sect. 2.1 and 4.1 we find $W_{e} \simeq 0.26 W_{i n}+0.28 W_{i n} \simeq 0.54 W_{i n}$, which is in agreement with the gradient found in Fig. 5 .

Thus also the linear relationship found by TEA is consistent with that expected by the MSM and the synthetic AL index. Even though the substorm energy output is assumed by construction to be proportional to the solar wind power input in the MSM, it is worth noting that the linear dependence is not an obvious consequence. In the MSM, the energy output depends on a single point measurement of the solar wind at the time of substorm onset, whereas in Fig. 5 the energy input integrates measurements over the expansion phase. Thus the linear dependence also stems from the long coherence in solar wind parameters relative to the expansion phase duration.

\subsection{Further work}

Whilst the MSM is able to account for the statistical distribution of substorm magnetic bay magnitude and waiting time, it is worth restating the caveat of $\mathrm{F}$ and $\mathrm{M}$ that it is uncertain to what extent the MSM can predict these variables for individual substorms. This is because (a) the solar wind power input driving the minimal model is estimated from singlepoint measurements taken several hundred $\mathrm{R}_{E}$ upstream of the earth and (b) the MSM is inherently non-linear as small uncertainties in the driving variables can cause large uncertainties in the simulated substorm onset time and magnitude. Importantly, these are factors that are likely common to most magnetospheric substorm models and so the question 
of predictability is quite generic. Work on this is currently in progress.

\section{Conclusions}

We have generated a synthetic AL index, using the Freeman and Morley (2004) minimal substorm model in conjunction with a simple model of ionospheric convection and some empirical relationships, from which model substorm bay magnitudes are derived. There are two free parameters in the model which scale the contributions to AL from the directly-driven DP2 electrojet and loading-unloading DP1 electrojet.

Comparison of the distribution of modelled substorm magnetic bay magnitudes, driven by $3.5 \mathrm{yrs}$ of solar wind measurements from the NASA Wind spacecraft, with 2 years of independent observations of substorm bay magnitudes made by TEA using the IMAGE magnetometer chain, shows that the minimal model can successfully reproduce the statistical distribution of this variable across a region of the 2-parameter space.

The ranges of the two parameters giving acceptable (5\% significance) agreement are consistent with expectations using results from other studies. The approximately linear relationship between the two free parameters over these ranges can be understood by a similar linear dependence of both directly-driven and unloading components on the solar wind power input, which is argued to be a valid approximation for certain conditions. For this approximation, the substorm magnitude simply scales linearly with the solar wind power input at the time of substorm onset such that $\mathrm{AL}_{p i}[\mathrm{nT}] \approx 48 P\left(t_{i}\right)\left[\mu \mathrm{W} \mathrm{m}^{-2}\right]$.

The linear relationship between energy input and energy output during the expansion phase previously observed by TEA is reproduced by the model (with similar variability). This supports an assumption of the model that the energy output is entirely determined by the state of the solar wind at substorm onset, but it is not a necessary consequence of this assumption. We conclude that the linearity is also due to the persistence (i.e. a long autocorrelation time) of the solar wind.

In the model, the Joule dissipation during the expansion phase is approximately $50 \%$ of the solar wind energy input during the same interval, which is higher than the observations of TEA. This discrepancy probably results from an overestimate of the fraction of total energy dissipated by Joule heating.

Acknowledgements. The authors thank the providers of the solar wind data used in this study: The ACE MAG and SWEPAM instrument teams and the ACE Science Center, the Wind MAG team (PI: R. Lepping) and Wind SWE team (PI: K. Ogilvie), and C. Perry (Rutherford Appleton Laboratory). We also thank G. Abel (British Antarctic Survey) for compiling the 1-min database from these data. SKM is the recipient of an Australian Research Council PostDoctoral Fellowship and is supported under the Australian Research
Council's Discovery funding scheme (Project DP0663643). MPF is supported by the BAS GSAC Natural Complexity programme.

Topical Editor I. A. Daglis thanks K. Kauristie and V. Sergeev for their help in evaluating this paper.

\section{References}

Ahn, B. H., Akasofu, S. I., and Kamide, Y.: The Joule heat production rate and the particle energy injection rate as a function of the geomagnetic indices AE and AL, J. Geophys. Res., 88, 6275-6287, 1983.

Akasofu, S. I.: The development of the auroral substorm, Planet.Space Sci., 12, 273-282, 1964.

Akasofu, S. I.: Magnetospheric substorms: a newly emerging model, Planet. Space Sci., 29 (10), 1069-1078, 1981.

Allen, J. H. and Kroehl, H. W.: Spatial and temporal distributions of magnetic effects of auroral electrojets as derived from AE indices, J. Geophys. Res., 80, 3667-3677, 1975.

Baker, D. N., Pulkkinen, T. I., Büchner, J., and Klimas, A. J.: Substorms: A Global Instability of the Magnetosphere - Ionosphere System, J. Geophys. Res., 104, 14 601-14 612, 1999.

Borovsky, J. E., Nemzek, R. J., and Belian, R. D.: The occurrence rate of magnetospheric-substorm onsets, in: Random and periodic substorms, J. Geophys. Res., 98, 3807-3814, 1993.

Caan, M. N., McPherron, R. L., and Russell, C. T.: The statistical magnetic signature of magnetospheric substorms, Planet. Space Sci., 26, 269-279, 1978.

Conover, W. J.: Practical Nonparametric Statistics, John Wiley, Hoboken, N. J., 3 edn., 1999.

Coumans, V., Gérard, J. C., Hubert, B., Meurant, M., and Mende, S. B.: Global auroral conductance distribution due to electron and proton precipitation from IMAGE-FUV observations, Ann. Geophys., 22, 1595-1611, 2004,

http://www.ann-geophys.net/22/1595/2004/.

Davis, T. N. and Sugiura, M.: Auroral electrojet activity index AE and its universal time variations, J. Geophys. Res., 71, 785-801, 1966.

Dungey, J. W.: Interplanetary magnetic field and the auroral zones, Phys. Rev. Lett., 6, 47-48, 1961.

Freeman, M. P.: A unified model of the response of ionospheric convection to changes in the interplanetary magnetic field, J. Geophys. Res., 108(A1), 1024, doi:10.1029/2002JA009385, 2003.

Freeman, M. P. and Farrugia, C. J.: A statistical study of the possible effect of solar wind variability on the recurrence rate of substorms, J. Geophys. Res., 100, 23 607-23 620, 1995.

Freeman, M. P. and Farrugia, C. J.: Solar wind input between substorm onsets during and after the October 18-20, 1995, magnetic cloud, J. Geophys. Res., 104, 22 729-22 794, 1999.

Freeman, M. P. and Morley, S. K.: A minimal substorm model that explains the observed statistical distribution of times between substorms, Geophys. Res. Lett., 31, L12807,doi: 10.1029/2004GL019989, 2004.

Freeman, M. P., Ruohoniemi, J. M., and Greenwald, R. A.: The determination of time-stationary two-dimensional convection patterns with single-station radar, J. Geophys. Res., 96, $15735-$ $15749,1991$.

Fukushima, N.: Equivalence in ground magnetic effect of Chapman - Vestine's and Birkeland - Alfvén's electric current systems for 
polar magnetic storms, Rep. Ionos. Space Res. Jpn., 23, 219-227, 1969.

Gjerloev, J. W. and Hoffman, R. A.: Height-integrated conductivity in auroral substorms 1. Data, J. Geophys. Res., 105, 215-226, 2000.

Hairston, M. R., Hill, T. W., and Heelis, R. A.: Observed saturation of the ionospheric polar cap potential during the 31 March 2001 storm, Geophys. Res. Lett., 30, 1325, doi:10.1029/2002GL015894, 2003.

Hill, T. W., Dessler, A. J., and Wolf, R. A.: Mercury and Mars: The role of ionospheric conductivity in the acceleration of magnetospheric particles, Geophys. Res. Lett., 3, 429-432, 1976.

Horton, W. and Doxas, I.: A low-dimensional dynamical model for the solar wind driven geotail-ionosphere system, J. Geophys. Res., 103, 4561-4572, 1998.

Ieda, A., Machida, S., Mukai, T., Saito, Y., Yamamoto, T., Nishida, A., Terasawa, T., and Kokubun, S.: Statistical analysis of plasmoid evolution with GEOTAIL observations, J. Geophys. Res., 103, 4453-4465, 1997.

Kallio, E. I., Pulkkinen, T. I., Koskinen, H. E. J., and Viljanen, A.: Loading-Unloading processes in the nightside ionosphere, Geophys. Res. Lett., 27, 1627-1630, 2000.

Kamide, Y. and Baumjohann, W.: Magnetosphere-Ionosphere Coupling, Springer-Verlag, New York, 1993.

Kauristie, K., Pulkinnen, T. I., Pellinen, R. J., and Opgenoorth, H. J.: What we can tell about auroral electrojet activity from a single meridional magnetometer chain, Ann. Geophys., 14, 1177-1185, 1996, http://www.ann-geophys.net/14/1177/1996/.

Klimas, A. J., Baker, D. N., Roberts, D. A., Fairfield, D. H., and Büchner, J.: A nonlinear dynamical analogue model of geomagnetic activity, J. Geophys. Res., 97, 12 253-12 266, 1992.

Klimas, A. J., Baker, D. N., Vassiliadis, D., and Roberts, D. A.: Substorm recurrence during steady and variable solar wind driving: Evidence for a normal mode in the unloading dynamics of the magnetosphere, J. Geophys. Res., 99, 14 855-14 861, 1994.

Klimas, A. J., Uritsky, V. M., Vassiliadis, D., and Baker, D. N.: Reconnection and scale-free avalanching in a driven current-sheet model, J. Geophys. Res., 109, A02218, doi: 10.1029/2003JA010036, 2004.

Koskinen, H. J. and Tanskanen, E. I.: Magnetospheric energy budget and the epsilon parameter, J. Geophys. Res., 107, 1415, 2002.

Lockwood, M. and Cowley, S. W. H.: Ionospheric convection and the substorm cycle, in "Substorms 1, Proceedings of the First International Conference on Substorms, ICS-1", ed C. Mattock, ESA-SP-335, European Space Agency Publications, Nordwijk, The Netherlands, 99-110, 1992.
Lui, A. T. Y.: A synthesis of magnetospheric substorm models, J. Geophys. Res., 96, 1849-1856, 1991.

Lui, A. T. Y.: A Multiscale Model for Substorms, Space Sci. Rev., 95, 325-345, 2001.

McPherron, R. L., Russell, C. T., and Aubry, M. P.: Satellite studies of magnetospheric substorms on 15 August 1968,9. Phenomenological model for substorms, J. Geophys. Res., 78, 3131-3149, 1973.

Morley, S. K. and Freeman, M. P.: On the association between northward turnings of the interplanetary magnetic field and substorm onsets, Geophys. Res. Lett., 34, L08104,doi:10.1029/2006GL028891, 2007.

Nishida, A. and Kokubun, S.: New polar magnetic disturbance, Rev. Geophys. Space Phys., 9, 417-425, 1971.

Perreault, P. and Akasofu, S. I.: A study of geomagnetic storms, Geophys. J., 54, 547, 1978.

Raeder, J. and Maynard, N. C.: Foreword, J. Geophys. Res., 106(A1), 345-348, 2001.

Reiff, P. H., Spiro, R. W., and Hill, T. W.: Dependence of polar cap potential drop on interplanetary parameters, J. Geophys. Res., 86, 7639-7648, 1981.

Ruohoniemi, J. M. and Greenwald, R. A.: Statistical patterns of high-latitude convection obtained from Goose Bay HF radar observations, J. Geophys. Res., 101, 21 743-21 763, 1996.

Russell, C. T., Luhmann, J. G., and Lu, G.: Nonlinear response of the polar ionosphere to large values of the interplanetary electric field, J. Geophys. Res., 106, 18 495-18 504, 2001.

Siscoe, G. L., Raeder, J., and Ridley, A. J.: Transpolar potential saturation models compared, J. Geophys. Res., 109, A09203, doi: 10.1029/2003JA010318, 2004.

Sun, W., Xu, W. Y., and Akasofu, S. I.: Mathematical separation of directly driven and unloading components in the ionospheric equivalent currents during substorms, J. Geophys. Res., 103, $11695-11700,1998$.

Tanskanen, E., Pulkkinen, T. I., Koskinen, H. E. J., and Slavin, J. A.: Substorm energy budget during low and high solar activity: 1997 and 1999 compared, J. Geophys. Res., 107, 1086, doi:10.1029/2001JA900153, 2002.

Viljanen, A. and Häkkinen, L.: Satellite-Ground Based Coordination Sourcebook, vol. ESA-SP-1198, chap. IMAGE magnetometer network, European Space Agency Publications, Noordwijk, The Netherlands, 111-117, 1997.

Weimer, D. R.: Substorm time constants, J. Geophys. Res., 99,11 005-11016, 1994. 